\title{
NORBERT WIENER ET L'ANALYSE DE FOURIER
}

\section{J.-P. KAHANE}

Il ne peut s'agir ici d'une revue complète de tout ce que Wiener a apporté à l'analyse de Fourier. Je voudrais seulement mettre l'accent sur son rôle de pionnier. Pour cela, quelques exemples suffiront.

1. La théorie générale des algèbres de Banach, élaborée par Gelfand, est souvent considérée aujourd'hui comme un fondement de l'analyse harmonique. Elle permet en tous cas d'aborder plus facilement, et de mieux comprendre, certaines questions comme les théorèmes taubériens de Wiener, dont parle ailleurs S. Mandelbrojt. Mais en vérité il n'est pas exagéré de dire que les algèbres de Banach sont issues du premier chapitre de Tauberian theorems [74]. ${ }^{1}$ L'étude qu'y fait Wiener des sommes de séries de Fourier absolument convergentes, et des transformées de Fourier de fonctions sommables sur la droite, a fourni un modèle aux travaux ultérieurs de Paul Lévy, de Beurling, et finalement de Gelfand. En même temps, les problèmes laissés en suspens ont stimulé des recherches pendant trente ans; rappelons seulement le célébre "problème de Wiener," implicitement posé par l'énoncé du Théorème III de Tauberian theorems: soit $f_{1}$ et $f_{2}$ deux fonctions sommables sur la droite; est-il vrai que $f_{2}$ soit limite dans $L^{1}$ de combinaisons linéaires de translatées de $f_{1}$ dès que sa transformée de Fourier $\hat{f}_{2}$ s'annule partout où $\hat{f}_{1}$ s'annule? En termes plus abstraits: est-il vrai que tout idéal fermé de l'algèbre de convolution $L^{1}(R)$ soit l'intersection des idéaux maximaux qui le contiennent? On sait que la réponse, négative, n'a été apporté qu'en 1959, par Malliavin (la réponse pour $L^{1}\left(R^{p}\right), p \geqq 3$, ayant été découverte antérieurement par L. Schwartz).

2. Autre modèle d'algèbre de Banach:l'algèbre $\hat{M}$ des transformées de Fourier-Stieltjes des mesures bornées sur la droite. Les idéaux maximaux n'y sont pas si simples que dans les exemples précédents; c'est ce que montre l'exemple de Wiener et Pitt [105]: une $\hat{\mu} \in \hat{M}$, telle que $1 / \hat{\mu}$ existe et soit bornée, mais n'appartienne pas à $\hat{M}$. Ç'a été là encore une belle source de travaux-le plus important restant le mémoire de Šreider de 1950.

3. Un sujet fascinant est la relation entre les propriétés d'une mesure bornée $\mu$ et le comportement à l'infini de sa transformée de

${ }^{1}$ Les nombres entre crochets renvoient à la bibliographie générale des oeuvres de Norbert Wiener. 
Fourier $\hat{\mu}$. Un fait fondamental découvert par Wiener, et d'ailleurs facile, est que la mesure $\mu$ est continue (c'est-à-dire que sa primitive est continue) si et seulement si $|\hat{\mu}|{ }^{2}$, ou aussi bien $|\hat{\mu}|$, a une valeur moyenne nulle sur la droite $[34],[73, \S 5]$. Avec Wintner [106], Wiener a construit d'autre part le premier exemple d'une mesure $\mu$ purement singulière telle que

$$
\hat{\mu}(u)=O\left(|u|^{-1 / 2+\varepsilon}\right) \quad(u \rightarrow \pm \infty, \epsilon>0 \text { donné }) ;
$$

la question était alors pendante depuis longtemps, et visiblement les auteurs ont eu une grande joie à la résoudre; ils ont même cru pouvoir construire la mesure $\mu$ de façon que son support soit un ensemble de mesure lebesguienne nulle, mais cela n'a été réalisé qu'ultérieurement, par Salem. Le problème est de savoir pour quelles fonctions $\phi(|u|)$ il existe une mesure $\mu$ purement singulière (ou mieux, une mesure $\mu \neq 0$ portée par un ensemble de mesure lebesguienne nulle), telle que $\hat{\mu}(u)=O(\phi(|u|))(u \rightarrow \pm \infty)$. Il n'a été, aussi complètement que possible, résolu qu'en 1957, par Ivašev-Musatov: si $\phi$ décroit assez régulièrement, la condition nécessaire et suffisante pour qu'il en soit ainsi est $\int_{0}^{\infty}|\phi|^{2}=\infty$.

4. Voici un autre aspect du sujet: quel est le comportement à l'infini de la transformée de Fourier $\hat{f}$ d'une fonction $f$ s'annulant en dehors d'un intervalle? Wiener développe cette question pour donner une nouvelle démonstration du théorème de Denjoy-Carleman sur les fonctions quasi-analytiques: la classe des fonctions $f$ telles que

$$
\sup _{x, n}\left|f^{(n)}(x) A_{n}^{-1}\right|<\infty
$$

est quasi-analytique si et seulement si

$$
\int_{0}^{\infty} \log \left(\sum_{n=0}^{\infty} \frac{x^{2 n}}{A_{n}^{2}}\right) \frac{d x}{1+x^{2}}=\infty \quad \text { ([92], Chapitre I). }
$$

En liaison justement avec l'introduction de nouvelles classes quasianalytiques et non quasi-analytiques (celles-ci étant destinées, par dualité, à fournir de nouvelles classes de distributions), le problème a été, au cours des dix dernières années, repris et étudié par Gelfand et Silov et par Mandelbrojt sous la forme suivante: pour quels couples de fonctions $\phi$ et $\psi$, assez régulières, existe-t-il une $f \neq 0$ telle que $|f| \leqq \phi$ et $|\hat{f}| \leqq \psi$ ? Encore qu'il ait beaucoup progressé récemment (les dernières contributions étant dues à Katznelson et Mandelbrojt), le sujet est loin d'être épuisé. 
5. Cela nous amène au cercle des idées développées dans le livre de Paley et Wiener: Fourier transforms in the complex domain [92]. D'abord le fameux théorème qui caractérise les transformées de Fourier des fonctions de carré sommable nulles hors d'un intervalle $[-a, a]$ comme fonctions de carré sommable qui sont restrictions à la droite réelle de fonctions entières de type exponentiel $\tau \leqq a$. Ce théorème est un outil très important pour de nombreuses questions d'analyse harmonique; outre celles, très nombreuses, qui figurent dans le livre de Paley et Wiener, ${ }^{2}$ signalons seulement la théorie des fonctions moyennes périodiques, sous la forme que lui a donné L. Schwartz en 1947. Il a été étendu à plusieurs dimensions et traduit en termes de distributions c'est sous cette forme, par exemple, qu'il intervient dans la démonstration du "théorème des supports" de Lions: étant donné deux distributions $S$ et $T$ à supports compacts, le plus petit convexe portant la convolution $S * T$ (qu'on appelle son support convexe) est la somme des supports convexes de $S$ et de $T$. Il a surtout attiré l'attention sur l'algèbre des fonctions entières de type exponentiel bornées sur la droite réelle, dont il convient de dire un mot maintenant.

6. Désignons par $E$ l'algèbre des fonctions entières de type exponentiel, par $E_{b}$ le sous-algèbre de $E$ formée des fonctions bornées sur la droite réelle, et notons $E^{p}$ et $E_{b}^{p}$ les sous-algèbres des précédentes formées de fonctions paires. On voit facilement que $E^{p}$ est l'ensemble des

$$
\begin{aligned}
f(z) & =\prod_{1}^{\infty}\left(1-\frac{z^{2}}{\lambda_{n}^{2}}\right), \quad \lambda_{n} \text { complexes, } \\
\lambda_{n} & =O(n) \quad(n \rightarrow \infty) .
\end{aligned}
$$

Ainsi le quotient de deux fonctions $\in E^{p}$, si c'est une fonction entière,

\footnotetext{
${ }^{2}$ Qu'on se référe pour cela à l'introduction, où Wiener célébre à juste titre la puissance de la méthode:

"this work covered a great variety of topics, but was unified by the central idea of the application of the Fourier transform in the complex domain. ... Nobody seems to have realized anything like the scope of the method. With its aid, we were able to attack such diverse analytic questions as those of quasi-analytic functions, of Mercer's theorem of summability, of Milne's integral equation of radiative equilibrium, of the theorems of Müntz and Szasz concerning the closure of sets of powers of an argument, of Titchmarsh's theory of entire functions of semi-exponential type with real negative zeros, of trigonometric interpolation and developments in polynomials of the form $\sum_{1}^{N} A_{n} \exp \left(i \lambda_{n} x\right)$, of lacunary series, of generalized harmonic analysis in the complex domain, of the zeros of random functions, and many others."
} 
appartient encore à $E^{p}$; et la même chose vaut pour $E$, d'après un théorème de Lindelöf. Mais le quotient de deux fonctions $\in E_{b}^{p}$ peut être une fonction entière sans appartenir à $E_{b}^{p}$ (par exemple, $z$ $\left.=\left(\sin ^{2} z / z\right)\left(\sin ^{2} z / z^{2}\right)^{-1}\right)$, et la caractérisation des suites $\left\{\lambda_{n}\right\}$ telles que (1) définisse une fonction $\in E_{b}^{p}$ est hors de portée. Faute d'étudier $E_{b}^{p}$, Paley et Wiener introduisent une algèbre intermédiaire entre $E_{b}^{p}$ et $E^{p}$, à savoir l'ensemble des fonctions (1) à zéros réels $\left(0<\lambda_{1}<\lambda_{2}<\cdots\right)$ satisfaisant l'une des propositions équivalentes qui suivent:

$$
\begin{gathered}
\log f(i y) \sim \pi A(y) \quad \text { quand } y \rightarrow \pm \infty, \\
\int_{-\infty}^{\infty} \log |f(x)| \frac{d x}{x^{2}}=-\pi^{2} A, \\
\lim _{n \rightarrow \infty} \frac{n}{\lambda_{n}}=A .
\end{gathered}
$$

La démonstration de l'équivalent $(2) \Leftrightarrow(3) \Leftrightarrow(4)$ occupe une place centrale dans le livre de Paley et Wiener (Chapitre V). Un peu plus tard, Levinson devait serrer de plus près l'étude de $E_{b}$, mais les progrès décisifs en la matière ne viennent que des travaux récents de Beurling et Malliavin, dont voici un résultat marquant: si $f \in E_{b}, g \in E_{b}$, et $h=f / g \in E$, il existe une $k \in E_{b}$, de type exponentiel arbitrairement petit, telle que $h k \in E_{b}$.

7. La question qui précède est en étroite relation avec la suivante: on donne une suite d'exponentielles $\left\{\exp \left(i \lambda_{n} t\right)\right\}$; il existe un $l \geqq 0$ tel que pour tout intervalle $I$ de longueur $<l$, ces exponentielles engendrent $L^{2}(I)$, et qu'il n'en soit plus ainsi dès que la longueur de $I$ dépasse $l$. Comment calculer $l$ quand on donne $\left\{\lambda_{n}\right\}$ ? Par dualité et transformation de Fourier, le problème revient à calculer la borne inférieure des types des fonctions $\in E_{b}$ qui s'annulent sur $\left\{\lambda_{n}\right\}$ $($ et $\neq 0)$.

Le cas $\lambda_{n}=i \mu_{n}, \mu_{n}>0$, étudié dans le Chapitre II du livre, avait déjà fait l'objet des travaux de Müntz et de Szasz: alors $l=0$ ou $l=\infty$ suivant que $\sum 1 / \mu_{n}$ converge ou diverge. Le plus intéressant est l'étude du sous-espace de $L^{2}(I)$ engendré par les exponentielles $\left\{\exp \left(-\mu_{n} t\right)\right\}$ lorsque $l=0$; le fait, fort intéressant, qu'il soit formé de fonctions analytiques a été découvert indépendamment aux alentours de 1940 par L. Schwartz, Clarkson et Erdös, et A. F. Leontiev.

Le cas où les $\lambda_{n}$ sont réels occupe tout le Chapitre VI, mais le calcul explicite de $l$ n'est fait que dans des cas particuliers. Ce n'est que dans le travail cité de Beurling et Malliavin que $l / 2 \pi$ apparait comme 
une "densitê" effectivement calculable ("densité effective extérieure").

8. Dans quel cas $\left\{\exp \left(i \lambda_{n} t\right)\right\} \quad n=\cdots-1,0,1, \ldots$ est-elle une base dans $L^{2}(-\pi, \pi)$ ? Pour donner au moins des conditions suffisantes, et comparer les développements suivant les bases $\{\exp (i n t)\}$ et $\left\{\exp \left(i \lambda_{n} t\right)\right\}$. Paley et Wiener introduisent (Chapitre VII) la notion de bases $\left\{f_{n}\right\}$ et $\left\{g_{n}\right\}$ d'un espace de Hilbert, voisines dans le sens que

$$
\sup \left\|\sum a_{n}\left(f_{n}-g_{n}\right)\right\|<1
$$

la borne supérieure étant prise pour toutes les suites complexes $a_{n}$ telles que

$$
\sum\left|a_{n}\right|^{2} \leqq 1 \text {. }
$$

C'est là le point de départ de nombreux travaux, et en particulier de ce que Duffin et Schaeffer ont appelé la théorie des cadres (1952).

9. L'étude des développements $\sum a_{n} \exp \left(i \lambda_{n} t\right)$ est évidemment en rapport avec la théorie des fonctions presque périodiques. Dans ce domaine, une nouvelle classe de fonctions a été introduite par Paley et Wiener (Chapitre VII) sous le nom de fonctions pseudopériodiques: $f$ est pseudopériodique si elle appartient à $L^{2}$ sur chaque intervalle borné et si les combinaisons linéaires de $f$ et de ses translatées ont des normes uniformément équivalentes dans $L^{2}(I)$ et $L^{2}(J)$ quand $I$ et $J$ sont des intervalles égaux assez grands; la borne inférieure des longueurs permises pour $I$ et $J$ est appelée la pseudopériode et notée $L(f)$. L'intérêt de cette notion est que, pour toute une foule de propriétés, le fait pour $f$ d'y satisfaire sur un intervalle de longueur supérieure à $L(f)$ entraine que $f$ y satisfait partout. Paley et Wiener montrent que $f$ est pseudopériodique si et seulement si $f$ est presque périodique au sens de Stepanoff avec un spectre régulier (c'est-à-dire inf $\left|\lambda_{m}-\lambda_{n}\right|=\delta>0$ ), et ils appliquent la méthode à des séries trigonométriques lacunaires (dans le sens $\lim _{n \rightarrow \pm \infty}\left(\lambda_{n+1}-\lambda_{n}\right)=\infty$ ). Un peu plus tard et indépendamment, Ingham a donné une inégalité sur les polynômes trigonométriques qui améliore l'estimation de la pseudopériode donnée par Paley et Wiener. Le calcul exact de la pseudopériode date d'une dizaine d'années, et fait intervenir la "densité uniforme extérieure" de la suite $\left\{\lambda_{n}\right\}$. Jusqu'il y a peu de temps, la notion de pseudopériodicité n'avait guère attiré l'attention; il y a pourtant là une méthode intéressante et une bonne réserve de problèmes. 
10. On connait mieux l'importante théorie que Wiener a développée sous le nom d'analyse harmonique généralisée; il s'agit essentiellement des fonctions mesurables $f$ telles que

$$
\phi(x)=\lim _{T \rightarrow \infty} \frac{1}{2 T} \int_{-T}^{T} f(x+u) \overline{f(u)} d u
$$

existe pour tout $x$ réel. Alors $\phi$ est transformée de Fourier d'une mesure positive, la mesure spectrale de $f$. Parmi elles figurent les fonctions presque périodiques de Bohr, mais aussi bien d'autres fonctions, en particulier les réalisations de nombreux processus stochastiques. Les idées développées dans le monumental article General harmonic analysis [73] ont, de ce fait, jeté un pont entre la théorie des processus stochastiques (mouvement brownien en particulier) et l'analyse de Fourier proprement dite. La portée des idées de Wiener en théorie des probabilités mérite une étude spéciale. Parmi celles, dans cet ordre de préoccupations, qui ont enrichi l'analyse harmonique, je me bornerai à un exemple [73, §12]. Pour démontrer l'existence d'une fonction $f$ dont la mesure spectrale soit continue, Wiener procède ainsi: il choisit au hasard avec la probabilité naturelle, indépendamment les uns des autres, des $\epsilon_{n}= \pm 1$, et pose $f(x)=\epsilon_{n}$ sur $[n, n+1[$; alors, presque sûrement, la mesure spectrale de $f$ est continue. Certes on peut construire des exemples explicites (Wiener en donne d'ailleurs un), mais la méthode introduite est générale et puissante, et elle a ensuite été appliquée dans des circonstances variées: au lieu de construire péniblement un contre-exemple, on construit un objet aléatoire dont presque toutes les réalisations constituent des contre-exemples.

Il faudrait encore signaler l'importance des idées de Wiener dans la théorie des équations intégrales (en particulier, celles du type de Wiener-Hopf); contentons nous de renvoyer à l'article de M. G. Krein paru dans les Uspehi en 1958.

Répétons qu'il ne s'agit là que de quelques exemples, destinés à dégager l'actualité de l'oeuvre de Wiener. La lecture des Selected papers [214] et des autres mémoires originaux, celle des livres de Wiener et en particulier du livre de Paley et Wiener, peuvent encore jouer un rôle de stimulant précieux en analyse de Fourier.

University OF Paris, PARIS, France 